\title{
Digital Control Method of Volume Clamp Method
}

\author{
Shouping Wang \\ Department of Electronic Science and Technology, \\ School of Information Science and Technology \\ University of Science and Technology of China \\ Hefei, China \\ e-mail: spw@mail.ustc.edu.cn \\ Jia Liu* \\ Shenzhen Institutes of Advanced Technology \\ Chinese Academy of Sciences \\ Shenzhen, China \\ The Chinese University of Hong Kong \\ Hong Kong, China \\ e-mail: jia.liu@siat.ac.cn \\ Pandeng Zhang \\ Shenzhen Institutes of Advanced Technology \\ Chinese Academy of Sciences \\ Shenzhen, China \\ e-mail:pd.zhang@siat.ac.cn
}

\author{
Xiaodan Yang \\ Shenzhen Institutes of Advanced Technology \\ Chinese Academy of Sciences \\ Shenzhen, China \\ e-mail: xd.yang@siat.ac.cn \\ Lu Huang \\ Department of Electronic Science and Technology, \\ School of Information Science and Technology \\ University of Science and Technology of China \\ Hefei, China \\ e-mail: luhuang@ustc.edu.cn
}

\begin{abstract}
The volume clamp method is a kind of non-invasive continuous blood pressure measurement methods, the traditional method is to convert light into a weak current to collect the signal of blood volume pulse by a sensor. In the process of signal transmission, it can be easily disturbed. We adopt a new method, the light is converted into frequency signal, by adopting a suitable method of frequency measurement, we can obtain blood volume pulse. This method improves anti-interference in the process of signal transmission, removes amplifier circuit and filter circuit. Meanwhile, this method also improves the measurement accuracy and real-time of system. On the other hand, due to removing $\mathrm{AD}$, we reduce the cost of system. At the end of this paper, we set up a hardware platform to respectively acquire blood volume pulse with the traditional method and our new method, the results show: the blood volume pulse with our new method is better than that with traditional method.
\end{abstract}

$\begin{array}{ll}\quad \text { Keywords-volume } & \text { clamp method; blood volume pulse; } \\ \text { anti-interference; } & \text { real-time performance;frequency } \\ \text { measurement } & \end{array}$

\section{INTRODUCTION}

The volume clamp method was by Penaz first suggested in 1973 [1]. Its theoretical basis is when the arterial wall is in a state of unloading due to external force, external pressure is equal to the arterial pressure. Therefore, according to the fluctuation of blood pressure, we can adjust external pressure to make arterial wall always in a state of unloading with quick response servo control system. By measuring the applied pressure, we can get the dynamic value of arterial blood pressure.

PPG system has at least one light source and at least one light detector, we can measure blood volume pulse of the finger by PPG system which is fed into a servo control system to drive proportional valve. When the blood vessel is controlled in a state of unloading, the counter pressure equals intra-arterial pressure [2][3][4]. But this method has a serious drawback, the current signal of the sensor output is very weak, it can be easily disturbed in the process of transmission. Therefore, there are some trouble for the hardware design and the software design.

In this paper, we adopt a new kind of sensor, the light is arranged into frequency signal which is transmitted to the servo control system to control the blood vessel in a state of unloading. This method has two advantages: 1 , strong anti-interference ability. 2, simplify the hardware circuit design. But the method need to adopt a kind of high precision frequency measurement method, we analyze several common square wave measurement methods and chose equal precision measurement method to measure the square wave frequency. 


\section{METHOD}

PPG (photo-plethysmographic) system is a typical kind of noninvasive measurement technique and method [5][6], which is based on biomedical sensor technology. Through the real-time tracing the optical absorption at the measured position (the finger, ear lobes, nose etc.) to obtain pulsatile change of peripheral microvascular volume. Clearly, here we need to keep current of LED constant in order to keep light intensity constant.

According to the different location of the sensors, the detection of blood volume pulse of the finger is divided into transmission type and reflection type [7]. Because the reflection type has a higher requirement for the location of LED and sensor, this article adopts the method of transmission type which is shown in Fig .1.

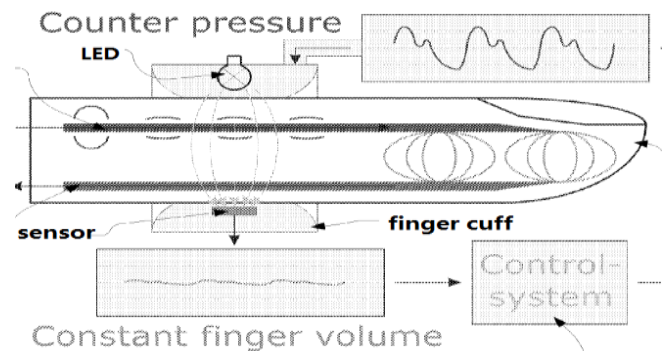

Figure 1. Transmission type

The sensor on opposite side of LED converts light through the finger into frequency signal which is transmitted to the servo control system to control the output frequency of PPG system to reach the set value. At this moment, the arterial wall is in unloading condition, we can get the counter pressure in finger cuff by pressure sensor, the counter pressure is equal to intra-arterial pressure.

\section{A. The Selection of LED's Wavelength [5]}

Fig .2 shows the light absorption rate of hemoglobin at different wavelengths. The absorption capacity of red blood cell in blood is a closely related to the state of the hemoglobin in blood, the light absorption coefficient of $\mathrm{HbO} 2$ and $\mathrm{Hb}$ at different wavelengths has evident difference. In the red light district, the absorption coefficient of $\mathrm{HbO} 2$ is much smaller than $\mathrm{Hb}$. In the infrared region, the absorption coefficient of $\mathrm{Hb}$ is less than $\mathrm{HbO} 2$. Because oxyhemoglobin is greater than hemoglobin in the artery, so we use infrared light. At the same time, in the 950nm wavelength, oxyhemoglobin absorption coefficient is higher, and near the wavelength, the absorption coefficient of oxyhemoglobin is almost constant, when the wavelength of LED has a little deviation, there is almost no influence for the absorption coefficient of oxyhemoglobin. Therefore, here we use a LED of 950nm wavelength.

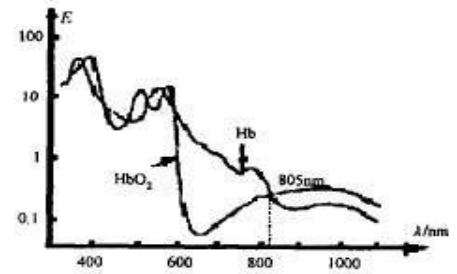

Figure 2. The light absorption curve of hemoglobin

source: http://www.hqew.com/tech/news/1101413.html

\section{B. The Choice of Sensor}

Here we choose TSL230RD which is a chip of TAOS. Its principle as shown in Fig .3.

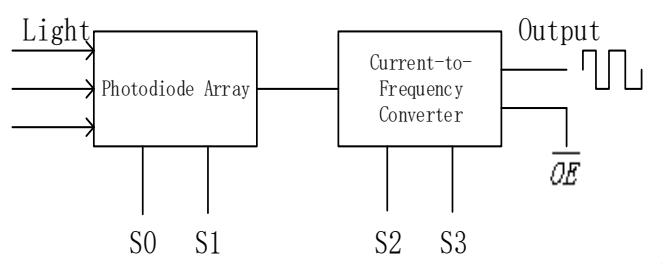

The principle of TSL230RD

Figure 3.

The received light signal can be converted into a square wave signal. At the same time, High and low level of the pin (S0, S1, S2, S3) can be set to adjust the range of square wave frequency and the size of light-sensing surface area. The chip pins show as follows:

\begin{tabular}{|cc|c|l|}
\hline \multicolumn{2}{|c|}{ TERMINAL } & TYPE & \multicolumn{1}{|c|}{ DESCRIPTION } \\
NAME & NO. & & \\
\hline GND & 4 & & Ground \\
\hline$\overline{O E}$ & 3 & I & Enable for $\mathrm{f}_{0}$ (active low) \\
\hline OUT & 6 & O & Scaled-frequency $\left(\mathrm{f}_{0}\right.$ ) output \\
\hline S0,S1 & 1,2 & I & Sensitivity-select inputs \\
\hline S2,S3 & 7,8 & I & $\mathrm{f}_{0}$ scaling-select inputs \\
\hline$V_{D D}$ & 5 & & Supply voltage \\
\hline
\end{tabular}

\section{Digital Frequency Measurement Methods [8]}

The digital frequency measurement methods commonly used have period measurement method, frequency measurement method and equal precision frequency measurement method. Period measurement method is suitable for measuring the high frequency signal, the higher frequency of measured signal, the smaller error. Frequency measurement method is suitable for measuring the low frequency signal, the lower frequency of measured signal, the higher measurement accuracy. Equal precision frequency measurement method can measure signal which has wider frequency range, and the measurement error is constant in the frequency range of the measured signal. The accuracy of the three methods as shown in Fig .4 [8], that the gate time is $5 \mathrm{~ms}$ and the frequency of reference signal is $60 \mathrm{MHz}$. 

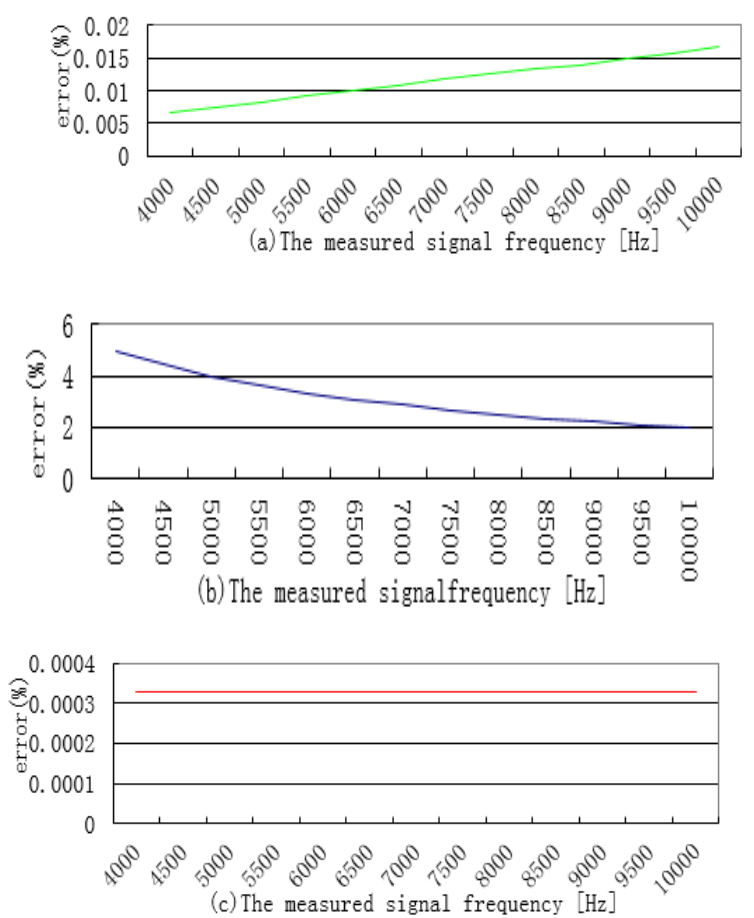

Figure 4. (a) period measurement method, (b) Frequency measurement method, (c) equal precision frequency measurement method

The frequency that is the output of PPG system is in the range of $4 \mathrm{~K} \sim 10 \mathrm{KHz}$. we can be seen from the Fig .4, equal precision frequency measurement method has the highest precision in the range, and its precision does not change with the change of the frequency. Therefore, this paper adopts equal precision frequency measurement method.

\section{The Principle of Equal Precision Frequency}

\section{Measurement Method}

The principle [9][10] of equal precision frequency measurement is shown in Fig .5.

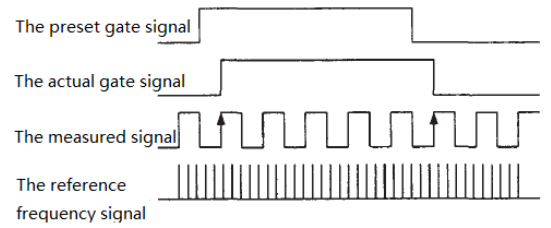

Figure 5. The principle of equal precision measuring method

In the process of measurement, there are two counters to count respectively for the standard signal and the measured signal at the same time. First of all, when the preset gate open signal comes (the preset gate signal rising edge), the counter does not begin to count, until the arrival of the rising edge of the measured signal, counter really began to count, then when the preset gate closed signal comes (falling edge), counter does not immediately stop counting, until the arrival of the rising edge of the measured signal, counter immediately stop counting and complete a measurement. The calculation formula of $\mathrm{F}_{\mathrm{X}}$ (the measured signal frequency) is as follows:

$$
\begin{aligned}
& \frac{F_{x}}{N_{x}}=\frac{F_{s}}{N_{s}}=\frac{1}{T_{c}} \\
& F_{x}=\frac{N_{x}}{N_{s}} * F_{S}
\end{aligned}
$$

Where Fx is the measured signal frequency, $\mathrm{Nx}$ is the number of the measured signal in the preset gate time, Fs is the frequency of reference signal, Ns is the number of reference signal in the preset gate time, Tc is the default gate time.

\section{E. Experiment data of blood volume pulse}

In order to compare the effect of the two methods for acquiring the blood volume pulse, we set up the hardware platform which is shown in Fig .6. As shown in Fig .8, the finger-cot contains finger cuff, senor and LED, the traditional method arranges light into current by sensor, which is transmitted to signal adjust circuit to amplify and filter and typed into Micro-Control Unit (MCU) through the AD sampling, our new method arranges light into frequency signal by a new sensor (for TSL230RD, $\mathrm{S} 0=\mathrm{S} 1=\mathrm{S} 2=1, \mathrm{~S} 3=0$ ), which is directly transmitted to Micro-Control Unit. In Fig .6, one finger-cot uses the traditional method, the other finger-cot uses our new method, two finger-cots are respectively wrapped in middle finger and index finger, as shown in Fig .7, and then we give two finger cuffs a same suitable pressure by pump with manual valve, we can know the value of their pressure by pressure gauge. After that, we respectively collect signal of blood volume pulse with traditional method and our method by data acquisition and calculation unit which contains signal adjust circuit, AD sampling and a micro-controller unit. The data that be collected is sent to computer and then through the DA of data acquisition card (USB 2811) to output. We can see the blood volume pulse by oscilloscope, as shown in Fig .9.

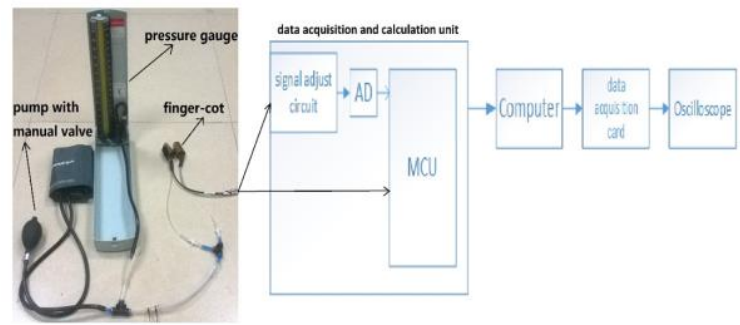

Figure 6. The hardware platform of acquiring the blood volume pulse

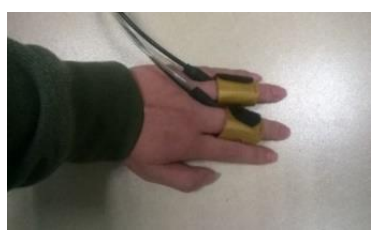

Figure 7. Finger-cots 


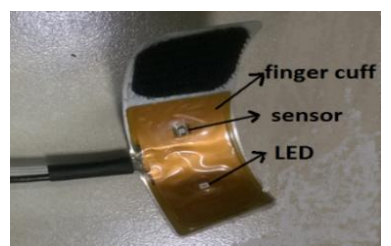

Figure 8. Components of finger-cot

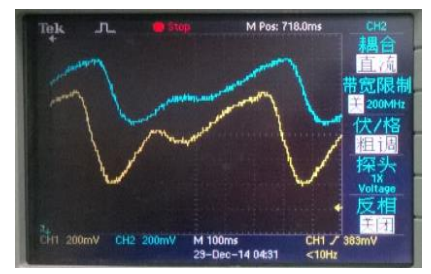

Figure 9. Blood volume pulse

In Fig .9, The blue curve represent blood volume pulse by the traditional method, the yellow curve represent blood volume pulse by our method. We can see from Fig .9: 1.Equal precision measuring method can measure the frequency of square wave very well. 2. Our method has a low time delay. 3. The traditional method has a weakening role for the high frequency component because of the filter.

\section{CONCLUSION}

Compared with traditional blood pressure measurement system of volume clamp method, our method has the follow advantages: 1 , our method reduces the cost of system because of removing AD. 2, because the signal of transmission is frequency signal, our method improves anti-interference in the process of signal transmission. 3, due to removing amplifier and filter circuit, our method improves the real-time performance of system. 4, the filter circuit removes the high frequency components of blood volume pulse, but our method removes filter circuit, it improves the accuracy of blood volume pulse.

\section{ACKNOWLEDGMENT}

This work was supported by a grant from Foshan City Collaborative Platform (2013HT100051), the work described in this paper was partially supported by the Project of Chinese Academy of Sciences and Shenzhen Science and Technology Project [Reference No. ZDS Y20120617113312191], National Natural Science Foundation of China (No. 81471207), Shenzhen Nanshan District Key Enterprises and Innovative Institution Supporting Funds (KC2014ZDZJ0022A) and the Guangdong Innovative Research Team Program (201001D0104648280)

\section{REFERENCES}

[1] Penaz J, "Photoelectric measurement of blood pressure, volume and flow in the finger," Digest of the 10th International Conferenceon Medical Engineering, vol. 104, 1973.

[2] Ben P.M. Imholz, Wouter Wieling, Gert A. van Montfrans, and Karel H. Wesseling, "Fifteen years experience with finger arterial pressure monitoring: assessment of the technology," Cardiovascular Research vol. 38, 1998, pp. 605-616.

[3] Roberto Maggi, Valentina Viscardi, Toshiyuki Furukawa, and Michele Brignole, "Non-invasive continuous blood pressure monitoring of tachycardic episodes during interventional electrophysiology," Europace, vol. 12, 2010, pp. 1616-1622.

[4] Wesseling KH, de Wit B, van der Hoeven GMA, van Goudoever J, and Settels JJ. Physiocal, "calibrating finger vascular physiology for Finapres," Homeostasis, vol. 36, 1995, pp. 76-82.

[5]Shi Ping, and Yu Hongliu, "Principles of photoplethysmography and its applications in physiological measurements," Journal of Biomedical Engineering, vol. 30, 2013, pp. 900-904 (in chinese).

[6] Giancarlo Fortino, and Valerio Giampà, "PPG-based Methods for Non Invasive and Continuous Blood Pressure Measurement: an Overview and Development Issues in Body Sensor Networks,' 978-1-4244-6290-2/10 @2010 IEEE.

[7] Wang Dongming, Zhang Song, Yang Yimin, and Li Xuwen, "Methods of non-invasive pulse wave detecting," Beijing Biomedical Engineering, vol. 29, 2010, pp.437-439 (in chinese).

[8] Xusheng Deng, "Analysis of four methods about frequency measurement by using single-chip Mircomputer-with the realization of equal accuracy method attached," Journal of test and measurement technique, vol. 10, 1996, pp. 391-396 (in chinese).

[9] Yu Yinghua, "Application of Equal Precision Frequency Measurement in the System of Computer on Slice," vol. 3, 1998, pp. 21-23 (in chinese).

[10]Li Guangming, and Yang Lei, "Method and Appication of Multi-period Frequency Measurement," Modern Electronic Technology, vol. 12, 2008, pp. 155-157. 